\title{
Research on Defensive Strategy Selection in Network Security
}

\author{
Hong $\mathrm{Li}$ \\ Sichuan Vocational College of Chemical Technology, \\ Sichuan 646005, china, \\ 99625057@qq.com
}

\begin{abstract}
Security analysis and attack-defense modeling are effective method to identify the vulnerabilities of information systems for proactive defense. The attack graph model reflects only attack actions and system state changes, without considering the perspective of the defenders. To assess the network information system and comprehensively show attack and defense strategies and theirs cost, a defense graph model is proposed. Compared with the attack graph, the model makes some improvements. Defense graph will be mapped to the attack and defense game model, in order to provide a basis for active defense policy decision. What's more, a generation algorithm of defense graph is proposed. A representative example is provided to illustrate our models and generation algorithm.
\end{abstract}

Keywords: Network Security, Active Defense, Attack-Defense Game Model

\section{Introduction}

Existing computer network information systems are deployed on multiple platforms [13], run numerous application software and support plentiful linkage patterns. Inevitably there will have some security vulnerabilities and faults. Network attacks tend to be diversified and integrated [4-5]. Due to limitations of resources and capability, network administrator can't eliminate drawbacks and defends all attacks neither. Network information security defenders always feel at loss and get in dilemma between information security investment and the best defense [6-18]. How to strike balance between information security risk and investment is a problem which needs consider defense cost effectiveness, making the most appropriate decisions with limited resources to reach "moderate security". That is a matter of selecting the optimal network security defense measures. Existing security model lacks description of the dynamic gaming between attack and defense, which can't reflect accurately the dynamic interaction and status evolution between attacker and defender in the system [19-28].

Here we talk about the complication of selecting the best defending strategies for network security. It defines formally the problem of choosing the best defensive strategies for network security, which are described with mathematical function [29-40]. Then, it gives the construction and solving method of selection function. Markov decision process has been successfully applied to search the optimal strategy by single intelligent agent under multiple states. Because of that, we can combine matrix type offensive and defensive game model and Markov decision process to get a random attack-defense game model which can be used to search dynamically the optimal attack-defense strategy by such a few rational intelligent agents under many offensive and defensive states. Random gaming [41-48] can be regarded as a state machine making game system shift from one status to the other with combined efforts of all players. Likewise, network system can be regarded as a state machine, which makes system change its status with co-action of offensive and defensive on target conflicts. The objective of attacker is to destroy the security attribute of network system. Defender prevents attacker from impairing secure 
attributes. System state change is not certain, moving from one state to another in a probability manner. Hence it's reasonable to depict network offence and defense conflict problem with random game model. In the static offense and defense game model, two sides search the best offensive and defensive strategy with static model in single state circumstances, no consideration of the dynamic state evolution or transition of offense and defense status. In this case, we propose random offensive and defensive game model which portrays the dynamic variations of network security offense and defense conflict and can solve the problem of selecting the best prevention strategy. The network attack and defense sides are modeled as a 2-person non-cooperative attack and defense random game model. It describes formalized definition and constituting elements of the model. Use attacker's privilege state on network entity components as element of offense and defense random game model; model the dynamic changes of network attack and defense states and predict attack behaviors and decide the best defending strategy. Next it presents the best defense strategy selection algorithm based on random offense and defense game model. Through discussion of network instances, we demonstrate the model and algorithm both work effectively on attack strategy prediction and optimal active defense [49-50].

\section{Formalized Description of the Optimal Defense Strategy Selection}

In network security management, the defender is perplexed by the defense strategy selection problem. What is the optimal defense strategy? A defensive strategy can reduce attack risk and loss but meanwhile defense cost is increased. How to weigh them and how to get the optimal defense strategy? The selection of strategy not only considers the cost and also network asset criticality and potential attack. For single attack, the defense decision needs choose a strategy which realizes the minimum defense cost; for multi-step and multiple attacks, some defending strategy is workable for one attack action but not for others. On that regard, we need consider the effectiveness of defense strategy for specific attack and the operation cost and negative influences. Besides, the happening probability of every attack strategy is unknown. How to guarantee defense tactics being the optimal and expected integrated defending cost being the lowest is a too complicated issue.

Based on above analysis, we can define the optimal defense strategy selection problem as one function: $f_{\text {selection }}=(C, A, D, G) \rightarrow D^{\prime}$; where each element is defined as follows:

$\mathrm{C}$ : entity component collection of protected network information system; entity component $c \in C$ can be host, router, firewall; each entity component is composed of part or more of software, hardware, data and service, e.g. Web server which runs HTTP service.

A: information aggregation of possible attack, happening attack and on the way. For network entity component $c \in C$, c has vulnerability which may be utilized by attacker; so it's required to recognize possible attack, occurrent attack and incoming attack $a \in A$ as to protect $\mathrm{C}$.

D: collection of defending strategies taken by defensive system, e.g. attack against Ftp Buffer overflow vulnerability; defending strategy $\mathrm{d}=($ close Ftp service),$d \in D$.

G: collection of offensive and defensive game models, such as matrix type offensive and defensive game model, random attack and defense game model ADSG introduced in next part, choose different game model according to specific network offensive and defensive situation.

$D^{\prime}$ : collection of optimal defending tactics taken by defense system, $D^{\prime} \subseteq D$

$f_{\text {selection }}$ is mapping from network security situation $(\mathrm{C}, \mathrm{A}, \mathrm{D}, \mathrm{G})$ to the optimal defense strategy $D^{\prime}$. For any $\mathrm{i}$, j, if $\left(C_{i}, A_{i}, D_{i}, G_{i}\right) \rightarrow D_{i}^{\prime},\left(C_{j}, A_{j}, D_{j}, G_{j}\right) \rightarrow D_{j}^{\prime}$, if $\left(C_{i}, A_{i}, D_{i}, G_{i}\right)=\left(C_{j}, A_{j}, D_{j}, G_{j}\right)$ then definitely $D_{i}^{\prime}=D_{j}^{\prime}$. Give network information 
system entity component collection $\mathrm{C}$; determine attack information aggregation $\mathrm{A}$ through vulnerability analysis and invasion detection mechanism; analyze $\mathrm{A}$ and $\mathrm{C}$ and determine defending strategy collection $\mathrm{D}$; choose suitable attack and defense game model $\mathrm{G}$ as per network attack-defense and requirement; with relative information about A, C, and D, construct the offense and defense game model G.

\section{Offense and Defense Random Game Model}

In this part we introduce matrix type offensive and defensive game model and Markov decision-making process. Markov decision process has been successfully used to search the optimal strategy by single intelligent agent under multiple states. By combining matrix type offense and defense game model and Markov decision-making process, we can have the random offense and defense game model used to dynamically find the best attackdefense strategy by many rational attack-defense intelligent agents in multiple offense and defense states.

\subsection{Matrix Type Attack-Defense Game}

Matrix type Attack-Defense Game (ADG) is a three tuple $\mathrm{ADG}=(\mathrm{N}, \mathrm{S}, \mathrm{U})$, where, 1. $N=\left(P_{1}, P_{2}, \ldots, P_{n}\right)$ is a set of players in the game of attack-defense. Participants are the main body of decision making and policy makers.

2. $S=\left(S_{1}, S_{2}, \ldots, S_{n}\right) \quad$ is a set of strategies for the attackdefense. $\forall i \in n, S_{i} \neq \varnothing . S_{i}=\left(S_{1}^{i}, S_{2}^{i}, \ldots, S_{m}^{i}\right)$ is the strategy set of offense and defense $P_{i}$. 3. $U=\left(U_{1}, U_{2}, \ldots, U_{n}\right)$ is a collection of the revenue function of the attack-defense. $\forall i \in n . U_{i}$ is the function of the offensive and defensive $P_{i}$.

\subsection{Markov Decision-Making Process}

Markov decision-making process is a dynamically optimized method built in the Markov process. Markov decision-making process has the basic thinking: transfer the state change of system to a Markov process to deal with; for every state in Markov process, decision-maker can take different actions according to various states and decision actions; decision-maker can gain certain revenue, which can be negative, meaning it suffers a loss. Markov decision process aims to find a strategy through which a proper decision action is taken in different states to make the ultimate benefit maximum or minimum. Markov decision process (MDP) [51] is a tetrad (, , A, T, R); S is state collection; A is system behavior collection; $\mathrm{T}$ is state transition function and $\mathrm{R}$ is revenue function. Mark $\mathrm{R}$ as profit value gained by system shifting state $\mathrm{s}$ to $s$ with the use of action a; mark $\mathrm{T}$ as probability of system moving state $\mathrm{s}$ to $s^{\prime}$ with the use of action a.

\subsection{Attack-Defense Random Game Model}

We extend single state of matrix type attack-defense. game model to multiple states and single intelligent agent in Markov decision-making process to many. By combining the two, we give out attack-defense random game model, defined as follows:

Definition: Attack-defense random game model (ADRG) is a 7 tuple $A D R G=\left(N, S, A, D, P, U_{a}, U_{d}\right)$, where,

$\mathrm{N}=\{$ Aattacker,Defender $\}$ is collection of players joining offense and defense game. If the number of attacker is $\geq 2$, it means distributive collaborative attack; if the number of defending system is $\geq 2$, it means collaborative protection by several defending system. Here we study when $n=2$, one attacker and one defender. Merge a couple of attackers and defenders and they can be seen as single attacker and defender. 
$S=\left(S_{1}, S_{2}, \ldots, S_{n}\right)$ A is an attack-defense random game state set.

$A=\left(a_{1} a_{2}, \ldots, a_{M}\right)$ is an attacker action set. An attacker in the game state $S_{k}$ attack action set $A_{k} \subset A$

$D=\left(d_{1}, d_{2}, \ldots d_{N}\right) \mathrm{A}$ is a set of defensive actions. The defender in the game state $S_{k}$ protective action set $D_{k} \subset D$

$P: S \times A \times D \times S \rightarrow[0,1]$ is the state transition probability function of the attackdefense random game.

$$
U_{K}: S \times A \times D \times S \rightarrow R, k=a, d \text { is a set of utility function. }
$$

The benefit relationship between attacker and defender includes zero-sum and nonzerosum. Choose zero sum or non-zero sum game model based on different network environment and attack-defense situation. In the course of network attack-defense game, the relationship between attacker and defender decides that either party won't notify the other of any strategy decision or they reach agreement. The relationship is always noncooperative and antagonistic. Therefore the above model is a non-cooperative attackdefense. random game model. Markov decision making process is single intelligent agent's multi-state model; matrix type attack-defense. game model is single-state model of multiple intelligent agents.

\subsection{Attack-defense. Random Game State}

We look network security state as attack-defense. random game state collection. Network security state describes network entity components' hardware and software resource attribute, connectivity, and user or attacker's ability to access the whole network. Security state change is caused by attack-defense. action pair $\left(a_{i}, d_{j}\right)$, in the form of file modification, system configuration variation, executable program running, attacker's privilege elevation etc. attack-defense random game state can be represented by a directed graph $G=(S, E)$, where $\mathrm{S}$ is graph's point set; each point represents a game state; $\mathrm{E}$ is collection of edges, meaning the transition relation of attack-defense. game state. Figure 1 illustrates game state and the transition relation by citing example of state graph which contains three game states $S=\left(S_{1}, S_{2}, S_{3}\right)$. In Figure1, state can mutually transit; but in some specific network environments, not all states can mutually transit. In it, $p_{i j}\left(a_{i}, d_{j}\right)$ marking between different states means the transitional possibility of game state changing from state $\mathrm{i}$ to state $\mathrm{j}$ under the action of attack-defense. strategy pair $\left(a_{i}, d_{j}\right)$.

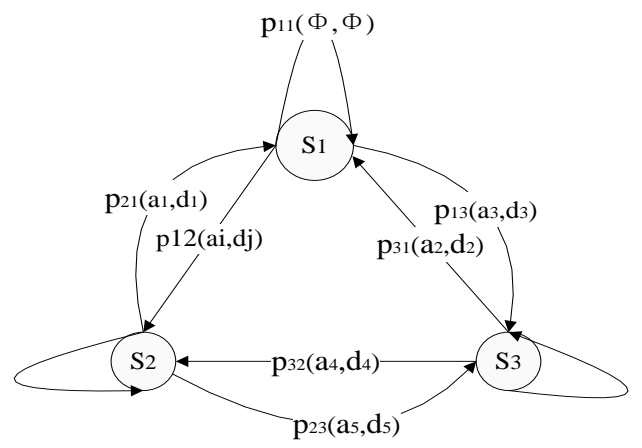

Figure 1. Example of ADRG's state 


\section{Defense Strategy Selection Algorithm based on Attack-Defense Random Game Model}

Given $(\mathrm{C}, \mathrm{A}, \mathrm{D}, \mathrm{G})$, where $G=A D R G=\left(N, S, A, D, P, U_{a}, U_{d}\right)$. Because there are many states in the game of attack-defense. Shapley iterative method can be used to solve the value $v=\left(v_{1}, v_{2}, \ldots, v_{k}\right)$ of offensive and defensive random game and its optimal attack-defense strategy. Defense strategy selection algorithm based on attack-defense random game model is as shown:

Algorithm1. Defense strategy selection algorithm based on attack-defense random game model Input: $(\mathrm{C}, \mathrm{A}, \mathrm{D}, \mathrm{G}), \delta$

Output: Optimal attack-defense strategy $\pi^{a^{*}}, \pi^{a^{*}}=D$

1. By C, A, D to build a random game of attack-defense $\mathrm{G}=\mathrm{ADSG}$

2. Arbitrary initial vector $v^{0}=\left(v^{0}(1), v^{0}(2), \ldots v^{0}(K)\right)$

3.Repeat

4.For every game state $S \in S_{k}$ do

5.For all $s_{i j}^{k} d o$

6. With $v_{l}$ instead of $s_{l}$

7.End for

8. Calculation $v_{k}^{r+1}=\operatorname{Val}\left(u_{i j}^{k}+\sum_{l=1}^{K} p_{i j}^{k l}\left(a_{i}, d_{j}\right) v_{l}^{r}\right)$

9.End for

10. For every game state $S \in S_{k} d o$

11. $v_{k} \leftarrow \operatorname{Val}\left(S_{k}\right)$

12. End for

13.Until $\left|v_{k}^{r+1}-v_{k}^{r}\right|<\delta, \forall S \in S_{k}$

14. For every game state $S \in S_{k} d o$

15. $\left(\pi_{k}^{a}, \pi_{k}^{d}\right)=\operatorname{Slove}\left(S_{k}\right)$

16.End for

17.Return $\pi^{a^{*}}=\left(\pi_{k}^{a}\right), \pi^{d^{*}}=\left(\pi_{k}^{d}\right)$

\section{Experimental Analysis and Results}

\subsection{Network Topology Structure}

Assuming that there is a typical network topology, the attack host is located in the external network, it is shown in Figure2. The protected network information system entity component set $\mathrm{C}=\{$ Attacker Host, Smtp Server, Ftp Server, Data Server $\}$ 。 


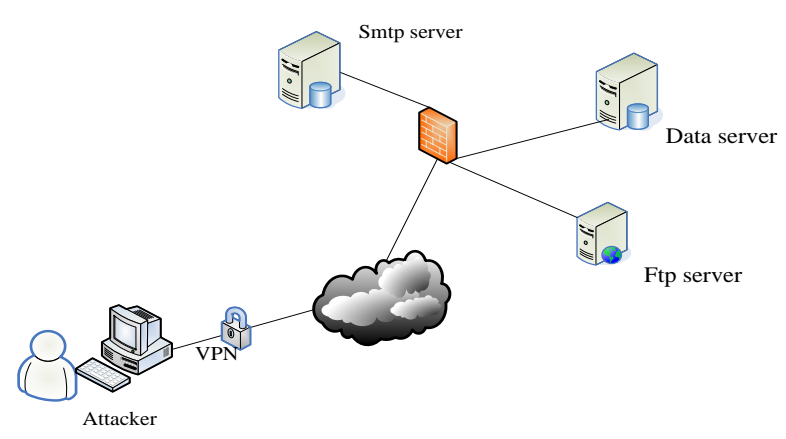

Figure 2. Topology of Example Network

The firewall separates the target network from external network, the firewall rules and the connection information are shown in Table 1.Target system vulnerability information is shown in table 2 .

Table 1. Connectivity in Example Network

\begin{tabular}{|l|l|l|l|}
\hline Source & Destination & Service & Action \\
\hline All & Smtp Server & Ftp, Smtp & Allow \\
\hline All & Ftp Server & Ftp, Ssh & Allow \\
\hline Smtp Server & Database & Oracle & Allow \\
\hline Ftp Server & Data Server & Oracle \\
\hline \multicolumn{4}{|c|}{ Table2 Vulnerability in servers } \\
\hline Host & Vulnerability & CVE ID & Allow \\
\hline Smtp Server & Ftp .rhost & $2011-0547$ & Effect \\
\hline Ftp Server & Ftp .rhost & $2011-0547$ & User privilege \\
\cline { 2 - 4 } & Sshd Buffer overflow & $2013-2421$ & User privilege \\
\hline Data Server & Oracle TNS Listener & $2012-0965$ & User privilege \\
\hline
\end{tabular}

\subsection{Description of Attack-Defense Random Game State}

Attacker's offense and control of the network are manifested in the control of network entity components, i.e. the acquisition of privilege by the attacker for entity components. We now introduce by expressing network security state as accessing ability of attacker to each network entity component, i.e. privilege; and network security state as attackdefense. random game state collection. Attack action leads to change of network security state, i.e. the state change of attacker's privilege for each component. Generally, we can define attacker's privilege state collection per actual network system environment. Here attacker's privilege state includes: no privilege, remote access privilege, local user privilege, and root privilege, which from low to high is no privilege<remote access privilege $<$ local user privilege $<$ root privilege.

Assume attacker has root privilege to invade host and launch attack but can't implement simultaneously multiple attack actions, with acquiring root privilege of Data Server as its goal. According to firewall rules, in Smtp Server and Ftp Server, attacker has the lowest remote access privilege and unable to access data server. However vulnerability exists in various components and they're dependent, attacker can utilize associated weakness to make multi-step attack and elevate privilege to get root privilege of Data Server, as described hereunder: in state $S_{1}$, attacker has root privilege to assault host; it can utilize respective vulnerability of Smtp Server and Ftp Server to launch Ftp.rhosts attach and obtain the local user privilege to arrive in state $S_{2}, S_{3}$. Since they're connected with Data Server, attacker uses them as springboard to employ Oracle TNS Listener's weak point to get Data Server's root privilege and reach state $S_{5}$. Besides, attacker can use Ftp Server's weakness Sshd Buffer overflow to make attack to get 
privilege of Ftp Server to reach state $S_{4}$; then, with the connection with Data Server, attacker utilizes Oracle TNS Listener's loophole to get root privilege of Data Server to reach state $S_{5}$. The directed graph $\mathrm{G}=(\mathrm{S}, \mathrm{E})$ is shown for attack-defense random game model. It is shown in Figure3.Game state $S=\left(S_{1}, S_{2}, S_{3}, S_{4}, S_{5}\right)$, each state is described as shown in Table 3.

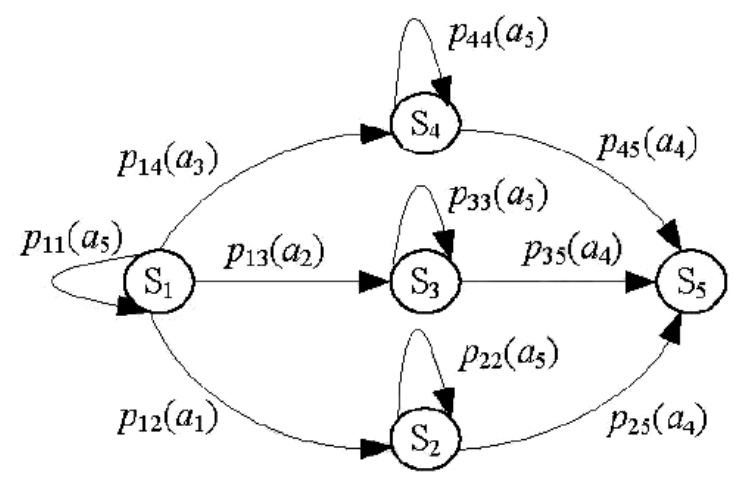

Figure 3. Attack-Defense Random Game State Graph

Table 3. Description of Random Game State

\begin{tabular}{|c|c|}
\hline State & Description \\
\hline$S_{1}$ & Root privilege on Attacker Host \\
\hline$S_{2}$ & User privilege on Ftp Server \\
\hline$S_{3}$ & User privilege on Smtp Server \\
\hline$S_{4}$ & User privilege on Ftp Server \\
\hline$S_{5}$ & Root privilege on Data Server \\
\hline
\end{tabular}

\subsection{Optimal Defense Strategy Selection}

With algorithm 1, we can solve out the state value of each attack-defense random game and the best attack-defense strategy, the value of each matrix type game model $\mathrm{Si}$ is calculated by Gambit method; finally the optimal attack-defense strategy is:

$$
\pi^{a^{*}}=\left(\pi_{1}^{a^{*}}, \pi_{2}^{a^{*}}, \pi_{3}^{a^{*}}, \pi_{4}^{a^{*}}\right), \pi^{d^{*}}=\left(\pi_{1}^{d^{*}}, \pi_{2}^{d^{*}}, \pi_{3}^{d^{*}}, \pi_{4}^{d^{*}}\right)
$$

In game state $S_{1}$, attacker takes the best offensive strategy: choose attack action $a_{1}$ in the probability 0.3 ; choose attack action $a_{2}$ in the probability 0.27 ; choose action $a_{3}$ in the probability 0.43 . In this state from the perspective of attacker, attack action $a_{1}, a_{2}, a_{3}$ works basically the same to the whole attack process, no big difference between cost and gain. For a3, the expectant revenue is high, so it's highly possible to take action $a_{3}$. For defender, the best defending tactics is: choose defensive action $d_{1}$ in the probability 0.39 , $d_{4}$ in 0.47 and $d_{6}$ in 0.14 . In the game state $S_{2}, S_{3}, S_{4}$, both take pure strategy, which conforms to the actual attack-defense. situation. For attacker, in game state $S_{2}, S_{3}, S_{4}$, there are only two options: attack or non-attack. Only the attack action is taken, can attacker achieve it purpose. So attacker makes attack in the probability 1. For defender, also in the state mentioned above, $d_{8}$ can yield the biggest revenue; therefore defender 
chooses it in the probability 1 . The expected return value of each game state is shown in table 4.

Table 4. Expected Payoff of Game State

\begin{tabular}{|c|c|c|c|c|c|}
\hline Game state & $S_{1}$ & $S_{2}$ & $S_{3}$ & $S_{4}$ & $S_{5}$ \\
\hline $\begin{array}{c}\text { Payoff of } \\
\text { Attacker }\end{array}$ & 364.5 & 64 & 64 & 64 & 40 \\
\hline $\begin{array}{c}\text { Payoff of } \\
\text { Defender }\end{array}$ & -364.5 & -64 & -64 & -64 & -40 \\
\hline
\end{tabular}

Figure 4 and Figure 5 describe the attack and defense strategy of the two sides in the game state $S_{1}$ probability change process. Gradually convergence tends to stable strategy to achieve the offensive and defensive Nash equilibrium.

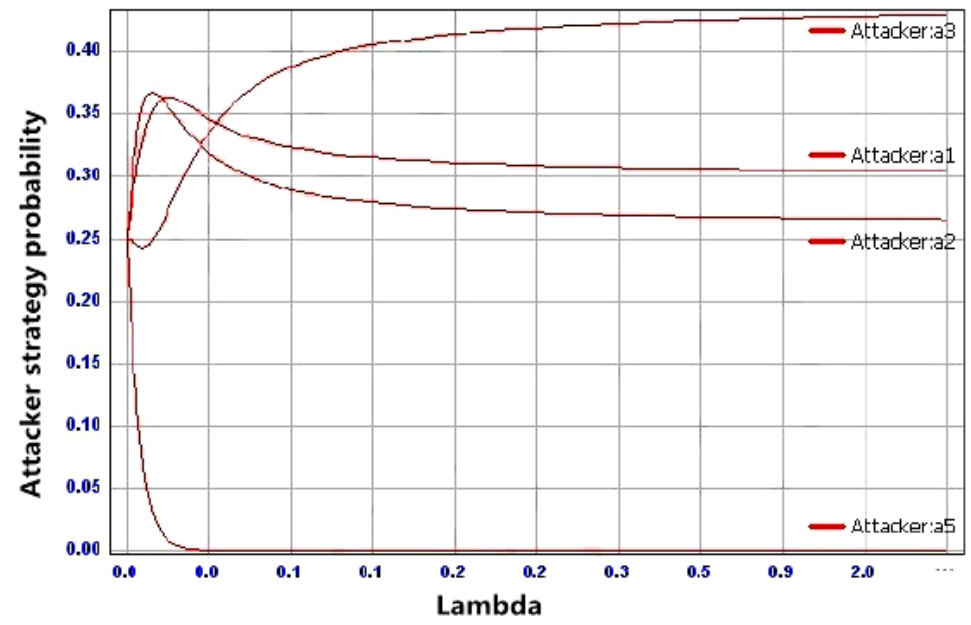

Figure 4. Probability of Attack Strategies in $S_{1}$

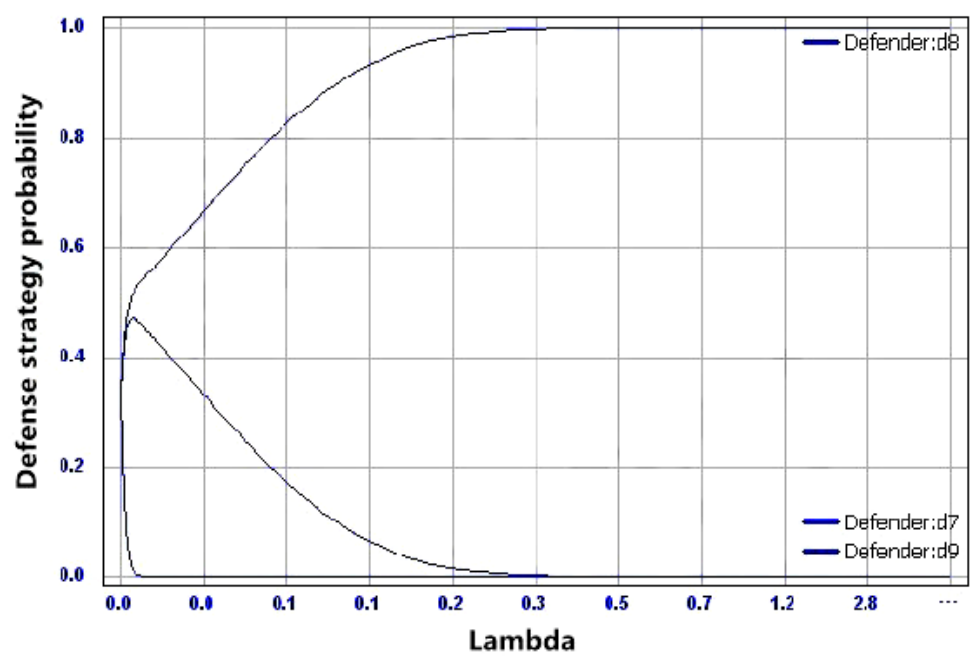

Figure 5. Probability of Defense Strategies in $S_{1}$

According to significances of Nash equilibrium in game theory, only defender sticks to its own Nash equilibrium strategy, may the system be impaired to the minimum extent during attack. The Nash strategy is obtained by analysis method of attack-defense. random game model provides solution to defender for active defense by selecting the 
optimal defending tactics. In actual application, security preventive measures can be taken based on network environment, security requirement and investment for security. If security prevention investment resource is limited, Nash equilibrium strategy is option. Moreover, properly increase defending investment to prevent all attack strategies as to reinforce network information system security.

\section{Conclusion}

In view of the complexity of network security optimal defense strategy selection, the formal definition of network security is the most selection of optimal defense strategy. In this paper, the static matrix model and the Markov decision process are extended, and the formal definition of a random game model is presented. The model can describe the dynamic change of network security attack and defense, in order to find the best offensive and defensive strategy for both offensive and defensive states. The experimental results show that the proposed model and algorithm are effective in attack strategy prediction and optimal active defense.

\section{References}

[1] F. Yu, Ch. Yongqiang, W. Song Yan, "Selection of network attack defense strategy based on a stochastic game model", Journal of Beijing University of Posts and telecommunications, (2014), S1, pp. 35-39.

[2] Z. Jian, Z. Hengwei, H. Jihong, "A method of defense strategy selection based on non cooperative game theory", computer science, (2016), 01, pp. 195-201.

[3] C. Yongqiang, W. Yu Fu and scutellarin, "Based on fuzzy static Bayesian game active network defense strategy selection", Application Research of computers, (2015), 03, pp. 887-889.

[4] Z. Jing, L. Yan, "Rough network security analysis model based on dynamic game. Computer Engineering", (2015), 04, pp. 129-134.

[5] C. Jianqiang, "Research on network vulnerability assessment based on game model", North China Electric Power University, (2011).

[6] W. Jindong, Y. Dingkun, Z. Hengwei, W. Na, "The selection method of active defense strategy of static Bias games", Journal of Xi'an Electronic and Science University, (2016), 01, pp. 144-150.

[7] L. Wangqun, W.Hui, Liu Jiahong, Deng Lei, Li Aiping, Wu Quanyuan, Jia Yan. Research on active defense technology of network security based on non cooperative dynamic game. Computer research and development, (2011),02, pp. 306-316.

[8] Y. tiequan, "The research and experiment of network attack and defense strategy and active defense based on game theory", Computer application and software, (2013), 09, pp. 312-315.

[9] W. Ying Han, Y. star, C. Yanmei, W. Xiuli, "Based on the game theory of enterprise information security defense strategy research", Computer engineering, (2013),09, pp.162-166

[10] L. Zhi, Hong, M. Chunlai, H. Jun, "Research on the selection of active defense strategy based on attack and defense in the network", The application of computer, (2015), 12 , pp. 3729-3734.

[11] J. Wei, "Research on the evaluation method of computer network vulnerability", University of Science \&amp; Technology China, (2012).

[12] N. Tong, "Research on Intrusion Forensics and active defense based on vulnerability correlation and game theory", Harbin Institute of Technology, (2012).

[13] L, Xiao: X. Chun Zhuang, W. Wen, "Based on stochastic game of network survivability strategy choice model", Computer applications, (2012),09, pp. 2609-2612.

[14] L S. Shapley, Stochastic games. Proceedings of the National Academy of Science USA, 1953,39: 1095 1100

[15] C. zhaoyuan, a new dynamic composite virtual network for active intrusion prevention. Northeast Forestry University, (2014).

[16] Z. Wei, "Research on Key Technologies of computer network defense strategy refinement", Beihang University, (2014)

[17] W. Wang. Research on the technology of distributed denial of service attack detection and defense. Northeastern University, (2011).

[18] W. Xinsheng. Research on Key Technologies of wireless sensor network intrusion tolerance. Jiangsu University, (2011).

[19] W. Chunzi. Research on Modeling and security evaluation of complex network attack. Xi'an University Of Architecture And Technology, (2011).

[20] T. Haokun. Under the dynamic environment of P2P worm defense model. University of Electronic Science and technology, (2013). 
[21] S. Chen. Research on key issues of wireless sensor network security based on game theory. Donghua University, (2013).

[22] Z. Feng. Research on active defense model of network security based on policy tree. Electronic Science and Technology University, (2004).

[23] Zhou. Research on network worm defense mechanism. technology P2P Chongqing University, (2007).

[24] Liang Xing. A network security access system based on active defense strategy. Huazhong University of Science and Technology, (2005).

[25] L. Juan Li. Research on network deception system based on game theory. Xi'an University Of Architecture And Technology, (2006).

[26] Z. Ying. "The study of active defense system" of Guizhou University, (2006).

[27] W. Zhiqiang. Vulnerability mining and related attack and Defense Technology Research Based on Fuzzy testing . Xi'an Electronic and Science University, (2015).

[28] Liu Gang, Li Qianmu, Zhang Hong. The generation method of network security defense strategy based on state attack defense graph model. computer application, (2013), S1, pp. 121-125.

[29] N. Tong. Research on Intrusion Forensics and active defense based on vulnerability correlation and game theory. Harbin Institute of Technology, (2012).

[30] L. Qun. Based on attack graph of network security independent decision-making. Jiangnan University, (2015).

[31] L. Zhiyan. Repeated game model of intrusion detection in wireless sensor networks. Jinan University, (2008).

[32] Y. Hongwei. Research and implementation of high performance intrusion detection technology based on multi core processor. Electronic Science and Technology University, (2009).

[33] M. ming. The abnormal phenomenon of cascading failure in complex Tien Lin power network fault analysis and defense. North China Electric Power University, (2014).

[34] B. Junrong. Research on network virus defense model based on game theory. North Central University, (2016).

[35] Gong park. Named non secure data network research of the defense strategy. Jiangsu University, (2016).

[36] Zhang Yejiang. Proactive P2P worm detection and defense technology research. Huazhong University of Science and Technology, (2009).

[37] L. Jibao. Network security situation awareness heterogeneous sensors on some key technologies. the Harbin Engineering University, (2009).

[38] S. Fei. Research on worm propagation modeling and defense strategy in the next generation network. Beijing University of Posts and Telecommunications, (2011).

[39] C. Gong. Research on network attack and security defense strategy. Journal of Sichuan University of Arts and Science, (2009), 02: pp. 26-29.

[40] X. Hui. Research on ecological defense strategy in the condition energy network. Shijiazhuang Railway University, (2013).

[41] Z. Hui. Campus network security model design based on intrusion detection system. Jilin University, (2004).

[42] W. Fei. Network security threats and defense strategy analysis. modern computer (Professional Edition), (2011),03, pp. 13-15.

[43] C. Zhaoyuan. Gnutella network worm propagation strategy and defense technology research. University of Electronic Science and technology, (2012).

[44] G. Wei, Y. Yu, "Deep defense strategy and implementation of campus network security", Journal of Jianghan University (NATURAL SCIENCE EDITION), (2007),02, pp. 60-64.

[45] Lijuan, "Cloud data security management strategy design", Dalian University of Technology, (2013).

[46] L. Peng. Analysis of computer network security defense strategy. computer CD-ROM software and applications, (2012),14, pp. 120-121.

[47] T. Yun, Z. Xuejun. Research on network scanning technology and security defense strategy. computer and digital engineering, (2008),04, pp. 90-93.

[48] Z. Weijun, Li Zhiwen. Analysis of the network information system in the information security defense resource allocation strategy based on the theory of constraints from the perspective. operations and management, (2014),03, pp. 163-169.

[49] L. Zhiwen, "The influence of herd behavior on information security investment strategy in network organization", science and technology management research, (2015), 16, pp. 170-174.

[50] Y. Xu, "Intrusion response decision model based on dynamic game theory", Jilin University, (2011).

[51] Howard, R. A. Dynamic programming and Markov processes. Cambridge, Massachusetts: The M.I.T. Press, (1960). 


\section{Author}

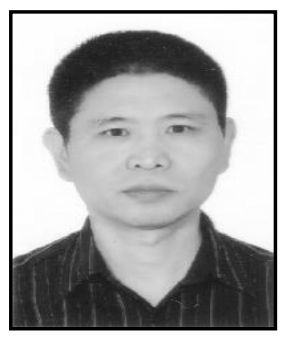

Hong Li. He received his B.S degree from Sichuan Normal University. $\mathrm{He}$ is an associate professor at Sichuan Vocational College of Chemical Technology. His research interests include software engineering, database and information system. 
International Journal of Security and Its Application Vol.11, No.1 (2017) 